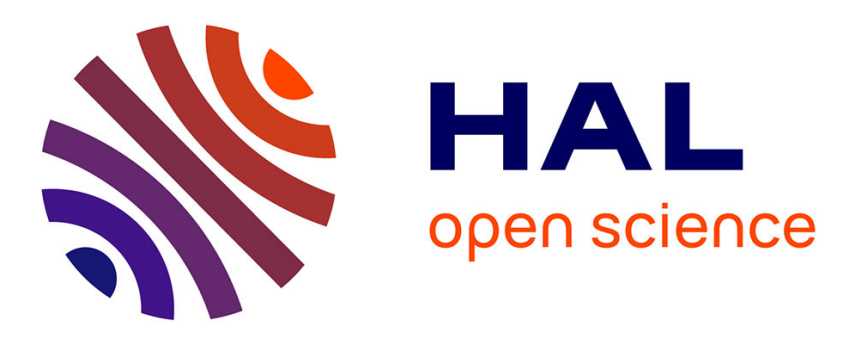

\title{
Validation of an all-sky imager-based nowcasting system for industrial PV plants
}

Pascal Kuhn, Bijan Nouri, Stefan Wilbert, Christoph Prahl, Nora Kozonek, Thomas C. Schmidt, Zeyad Yasser, Lourdes Ramírez, Luis Zarzalejo, Angela Meyer, et al.

\section{To cite this version:}

Pascal Kuhn, Bijan Nouri, Stefan Wilbert, Christoph Prahl, Nora Kozonek, et al.. Validation of an all-sky imager-based nowcasting system for industrial PV plants. Progress in Photovoltaics, 2017, Online Version of Record published before inclusion in an issue. 10.1002/pip.2968 . hal-01667733

\section{HAL Id: hal-01667733 \\ https: / hal-mines-paristech.archives-ouvertes.fr/hal-01667733}

Submitted on 21 Dec 2017

HAL is a multi-disciplinary open access archive for the deposit and dissemination of scientific research documents, whether they are published or not. The documents may come from teaching and research institutions in France or abroad, or from public or private research centers.
L'archive ouverte pluridisciplinaire HAL, est destinée au dépôt et à la diffusion de documents scientifiques de niveau recherche, publiés ou non, émanant des établissements d'enseignement et de recherche français ou étrangers, des laboratoires publics ou privés. 


\title{
Validation of an all-sky imager-based nowcasting system for industrial PV plants
}

\author{
Pascal Kuhn ${ }^{1}$ (1) | Bijan Nouri ${ }^{2}$ | Stefan Wilbert ${ }^{2}$ | Christoph Prahl ${ }^{2}$ | Nora Kozonek ${ }^{2}$ | \\ Thomas Schmidt $^{3}$ | Zeyad Yasser ${ }^{4}$ | Lourdes Ramirez ${ }^{5}$ | Luis Zarzalejo ${ }^{5}$ | Angela Meyer ${ }^{6}$ | \\ Laurent Vuilleumier $^{6}$ | Detlev Heinemann ${ }^{7}$ | Philippe Blanc ${ }^{8}$ | Robert Pitz-Paal ${ }^{9}$
}

${ }^{1}$ Institute of Solar Research, Researcher, M.Sc. Physics, German Aerospace Center (DLR), Plataforma de Almería, Ctra. de Senés s/n km 5, 04200 Tabernas, Spain

${ }^{2}$ Institute of Solar Research, German Aerospace Center (DLR), Plataforma de Almería, Ctra. de Senés s/n km 5, 04200 Tabernas, Spain

${ }^{3}$ CSP Services GmbH, Friedrich-Ebert-Ufer 30, 51143 Cologne, Germany

${ }^{4}$ TSK FLAGSOL, Anna-Schneider-Steig 10, 50678 Cologne, Germany

${ }^{5}$ Energy Department, Renewable Energy Division, CIEMAT, Av. Complutense, 40, 28040 Madrid, Spain

${ }^{6}$ MeteoSwiss, Les Invuardes, 1530 Payerne, Switzerland

${ }^{7}$ Energy Meteorology Unit, Energy and Semiconductor Research Laboratory, Institute of Physics, Oldenburg University, 26111 Oldenburg, Germany

${ }^{8}$ O. I. E.-Centre Observation, Impacts, Energy, MINES ParisTech, PSL Research University, CS10207, F-06904 Sophia Antipolis CEDEX, France

${ }^{9}$ Institute of Solar Research, German Aerospace Center (DLR), Linder Höhe, 51147 Cologne, Germany

Correspondence

Pascal Kuhn, German Aerospace Center (DLR), Institute of Solar Research, Plataforma de Almería, Ctra. de Senés s/n km 5, 04200

Tabernas, Spain

Email: pascal.kuhn@dlr.de

Funding information

Bundesministerium für Wirtschaft und Energie, Grant/Award Number: 0325848A; European Union's FP7 programme, Grant/ Award Number: 608623; European Union's Horizon 2020 programme, Grant/Award Number: 654984; German Federal Ministry for Economic Affairs and Energy within the WobaS project

\begin{abstract}
Because of the cloud-induced variability of the solar resource, the growing contributions of photovoltaic plants to the overall power generation challenges the stability of electricity grids. To avoid blackouts, administrations started to define maximum negative ramp rates. Storages can be used to reduce the occurring ramps. Their required capacity, durability, and costs can be optimized by nowcasting systems. Nowcasting systems use the input of upward-facing cameras to predict future irradiances. Previously, many nowcasting systems were developed and validated. However, these validations did not consider aggregation effects, which are present in industrial-sized power plants. In this paper, we present the validation of nowcasted global horizontal irradiance (GHI) and direct normal irradiance maps derived from an example system consisting of 4 all-sky cameras ("WobaS-4cam"). The WobaS-4cam system is operational at 2 solar energy research centers and at a commercial 50-MW solar power plant. Besides its validation on 30 days, the working principle is briefly explained. The forecasting deviations are investigated with a focus on temporal and spatial aggregation effects. The validation found that spatial and temporal aggregations significantly improve forecast accuracies: Spatial aggregation reduces the relative root mean square error (GHI) from $30.9 \%$ (considering field sizes of $25 \mathrm{~m}^{2}$ ) to $23.5 \%$ (considering a field size of $4 \mathrm{~km}^{2}$ ) on a day with variable conditions for 1 minute averages and a lead time of 15 minutes. Over 30 days of validation, a relative root mean square error (GHI) of $20.4 \%$ for the next 15 minutes is observed at pixel basis $\left(25 \mathrm{~m}^{2}\right)$. Although the deviations of nowcasting systems strongly depend on the validation period and the specific weather conditions, the WobaS-4cam system is considered to be at least state of the art.
\end{abstract}

\section{KEYWORDS}

all-sky imager, grid integration, nowcasting systems, photovoltaic, plant control, solar nowcasting 


\section{1 | INTRODUCTION, AIM, AND APPROACH}

The solar resource shows variabilities, which influence the frequency, voltage, and overall stability of electrical grids with high penetrations of solar power plants. Facing this challenge, legal limitations of the fluctuations of generated electricity (ramp rates) are already effective or being discussed. ${ }^{1}$ Unsurprisingly, small island grids with high solar penetration had to rush ahead: Hawaii has defined a maximum negative ramp rate of $1 \mathrm{MW} / \mathrm{min}$ during certain times, ${ }^{1}$ and Puerto Rico has specified a maximum negative 1 -minute ramp rate of $10 \%$ of the rated capacity. ${ }^{2}$ For short-term periods between 0 and 30 minutes, the origins of these ramp rates for photovoltaic (PV) plants are predominantly transient clouds. Forecasts covering this period are called nowcasts and can be achieved by all-sky imager-based systems. ${ }^{3-11}$ By providing such forecasts, nowcasting systems can help the power plant to fulfill these ramp rate regulations. Moreover, they help to optimize the required size and the operations of combined PV-battery systems. ${ }^{12,13}$ Thus, optimized operations potentially increase the lifespan of electrical storages and auxiliary devices. ${ }^{14}$

The 4-camera WobaS system (WobaS-4cam), which is presented in this work, is installed at PSA (Plataforma Solar de Almería, Spain). ${ }^{11}$ WobaS stands for the research project "Wolkenkamera-basierte Betriebsstrategien für Solarkraftwerke"-all-sky imager-based operational strategies for solar power plant. Another WobaS-4cam system operates at the commercial solar power plant La Africana (near Córdoba, Spain), ${ }^{15}$ and a third WobaS-4cam system is running at a solar research center of the University of Evora, Portugal. ${ }^{16}$ Further, WobaS systems based on 1 and 2 camera configurations have been developed (not presented here). WobaS-4cam systems use the inputs of 4 off-the-shelf surveillance cameras (Mobotix Q24 or Q25). Besides their low costs, these cameras were found to be easy to operate and reliable in harsh weather conditions. Synchronized by an NTP server, the cameras take images every 30 seconds with resolutions of $3 \mathrm{MP}$ (Mobotix Q24) or 6 MP (Mobotix Q25). In these images, clouds are segmented using a 4-dimensional clear sky library (CSL). ${ }^{11}$ The 3-D positions and shapes of all visible clouds are determined via voxel carving. ${ }^{17}$ Voxel is an abbreviation for volume element, and voxel carving is the process to derive 3-D objects out of multiple camera perspectives. This way, a voxel space filled with individual cloud objects is derived (Figure 1, left). Since the ground cameras cannot see on the top of the clouds (self-occlusion), the raw cloud voxels must be partially modelled.
By tracking 3-D cloud objects over multiple timestamps, cloud velocities and directions are derived and used to predict cloud movements. Future cloud positions are calculated for lead times up to 15 minutes ahead. With the sun position and the surface elevations known, the shadows on the ground are deduced. ${ }^{17}$ If available, reference real-time irradiance measurements are used to determine cloud transmittances. With cloud transmittances derived, shadow maps are transformed into irradiance maps (see Figure 1, right). WobaS systems can use ground measurement stations for direct normal irradiance (DNI) and global horizontal irradiance (GHI). Without available ground measurements, modelled irradiance data can be used.

The validated WobaS-4cam system is located at PSA and has access to DNI, GHI, and DHI (diffuse horizontal irradiance) measurements and is able to predict GHI, DNI, and GTI (global tilted irradiance) maps. Spatial and temporal resolutions for these maps are $25 \mathrm{~m}^{2}$, updated every 30 seconds for predictions up to 15 minutes ahead. The working principle of the WobaS system is illustrated in Figure 2.

This publication is structured as follows: In Section 2, as an example for the validation of a subtask, the cloud detection within all-sky images is presented. The validation of nowcasted irradiance maps is presented in Section 3. The focus of this validation lies on the investigation of spatial and temporal aggregation effects. Spatial aggregation effects are relevant for large solar plants, covering several square kilometers: Since the aggregated total electricity production of the PV plant is the most important parameter to forecast, the accuracies and validations of the average nowcasted irradiances over the complete plant area are of special interest. The validation of the nowcasted irradiance maps is conducted via pyranometer $(\mathrm{GHI})$ and pyrheliometer (DNI) measurements (Section 3.1) and with a reference shadow camera system (Section 3.2). The conclusion is given in Section 4.

\section{2 | VALIDATION OF CLOUD SEGMENTATION}

As an example for the validation of a subtask, the validation of a cloud detection approach is discussed in this section. Validations of other subtask, for instance, the derivation of cloud heights ${ }^{18}$ and cloud speeds, ${ }^{19}$ are presented elsewhere.

Segmenting clouds in all-sky images is one key task of the nowcasting system and is surprisingly difficult. This originates from color and intensity dependencies within the images regarding pixel
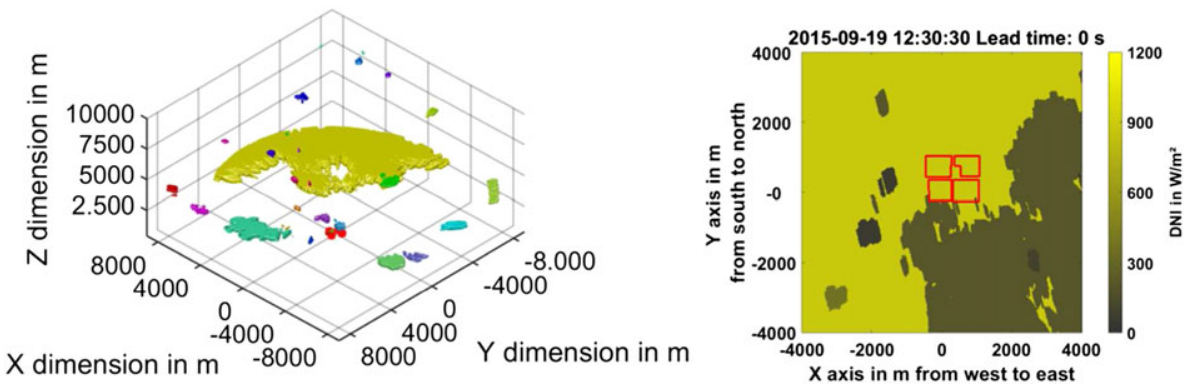

FIGURE 1 Several cloud objects in the voxel space (left) and corresponding nowcasted direct normal irradiance (DNI) map (right) at the La Africana solar power plant [Colour figure can be viewed at wileyonlinelibrary.com] 


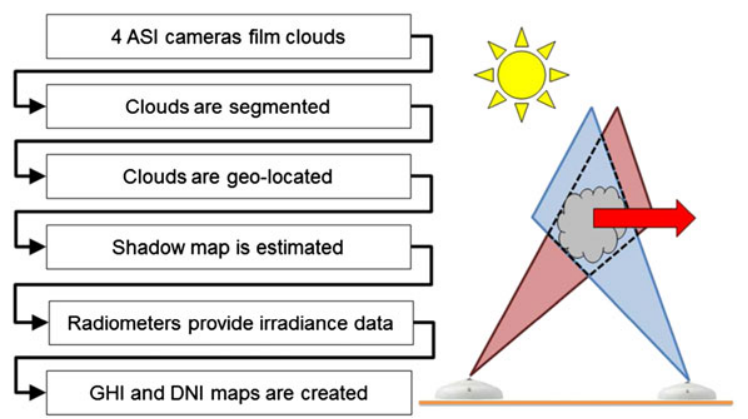

FIGURE 2 Working principle of the WobaS all-sky imager-based nowcasting system. All-sky imagers (ASIs) are used to detect, 3-D geo-locate, and track clouds to predict cloud shadows on the ground. This way, future irradiances in high spatial and temporal resolutions can be derived [Colour figure can be viewed at wileyonlinelibrary.com]

and sun positions as well as from artefacts due to saturation and glare effects in the circumsolar region. Many approaches, eg, based on neural networks ${ }^{20,21}$ or ratios of the red-green-blue (RGB) color channels, ${ }^{22,23}$ are validated and discussed in the literature. The WobaS-4cam nowcasting system uses a 4-dimensional CSL. ${ }^{11}$ In the CSL, clear sky RGB values for every pixel depending on sun pixel angle (SPA), the pixel zenith angle (PZA), air mass (related to solar elevation for a given altitude), and Linke turbidity are stored. The 2 angles are illustrated in Figure 3A: The PZA is the angle between the zenith above the camera and the pixel in the fisheye projection. The SPA is the angular distance between the positions of the sun and the pixel.

The Linke turbidity is calculated from DNI or $\mathrm{GHI}$ measurements using the Linke turbidity model from. ${ }^{24}$ Via the temporal variation of the Linke turbidity, shaded ground measurements are excluded using a Linke turbidity-based method presented in the previous studies. ${ }^{25,26}$ Afterwards, the current Linke turbidity is determined using a linearly time-weighted average of the most recent Linke turbidities derived from unshaded irradiance measurements.

The CSL consists of 80 layers per color channel. Each layer corresponds to a specific jointed air mass and Linke turbidity bin. Within a layer, the RGB values of images taken during clear sky conditions are stored relative to 2 angles (SPA, PZA; see Figure 3A). In Figure 3B, 8 layers of the red-to-blue ratio are depicted, which are calculated from the corresponding color channel layers.
If a pixel in the image under consideration is clouded, its RGB values deviate from the CSL, and this way a cloud is detected. The detection is based on CSL color channel ratios (as depicted in Figure 3 B) or differences and situational thresholds. Figure 4 illustrates the approach in a simplified manner: From the raw image, ratios and difference of the color channels are calculated. On the basis of the current air mass and Linke turbidity, 1 CSL layer per color channel is selected. From these CSL layers, ratios (depicted: red-to-blue ratio) and differences are calculated and compared to the current image. Using multiple thresholds, cloud detection is then performed. The thresholds used for the segmentation depend on PSA, PZA, and on the current weather situation (eg, overcast, clear sky). The current weather situation is determined considering irradiance and illuminance fluctuations, detected movements, and cloud coverages found in the previous segmentations.

The CSL-based segmentation is validated with approximately 600 manually segmented images per camera model. The approach is illustrated in Figure 5 for the Mobotix Q24 camera model, for which a total of 612 reference images for various situations were manually segmented. In this approach, all-sky images are automatically segmented by the algorithm and pixel-wise compared to manually segmented references.

In Figure 6, the results of the validation on 460 Mobotix Q24 images are presented for several weather situations divided into groups with high (above $30^{\circ}$ ) and low sun elevation angles (below $20^{\circ}$ ), hazy (Linke turbidity above 3.25 ) and less hazy conditions (Linke turbidity below 2.75) as well as different cloud coverages. Further, 152 manually segmented reference images are available to validate specific approaches for the circumsolar area (not presented). The cloud coverages are categorized into clear sky situations with less than $2.5 \%$, scattered situations with coverages between $2.5 \%$ and $80 \%$, and (nearly) overcast situations with more than $80 \%$ cloud coverage. Clear sky situations are defined to have cloud coverages below $2.5 \%$, not exactly $0 \%$, as the field of vision of an all-sky imager includes objects with heights of $10 \mathrm{~km}$ at distances of more than $50 \mathrm{~km}$ (Figure 10B). Situations with exactly $0 \%$ cloud coverage inside an all-sky image are thus rare, even in southern Spain.

The 3 cloud coverage categories ("clear sky," "scattered," "overcast") were chosen as segmentation approaches might detect the sun or the circumsolar area, especially for high Linke turbidities, incorrectly as clouds during clear sky situations and simple
FIGURE 3 A, Imaged semi-dome of the all-sky imager with relevant angles of the clear sky library (CSL) marked. B, Layers of the CSL for a Linke turbidity range between $T L=1$ and $T L=$ 2.3 and the air masses between 0 and 10. In the CSL, a total of 80 layers per color channel for various Linke turbidities and air masses are stored. RBR, red-to-blue ratio [Colour figure can be viewed at wileyonlinelibrary.com]

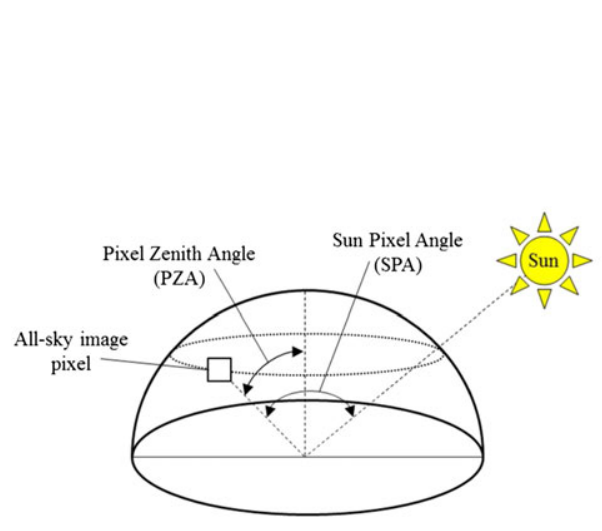

(a)

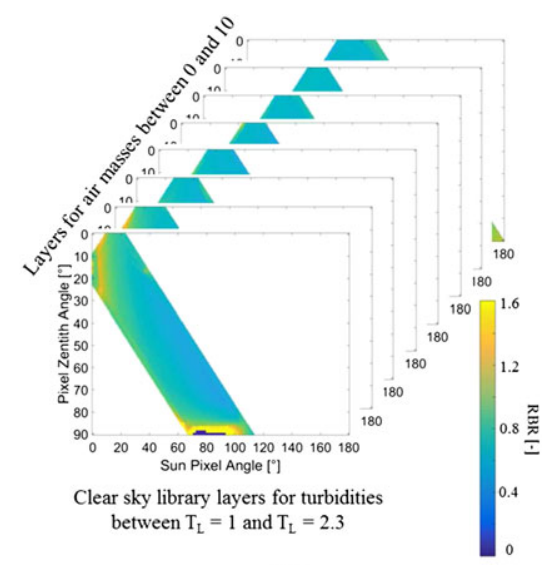

(b) 

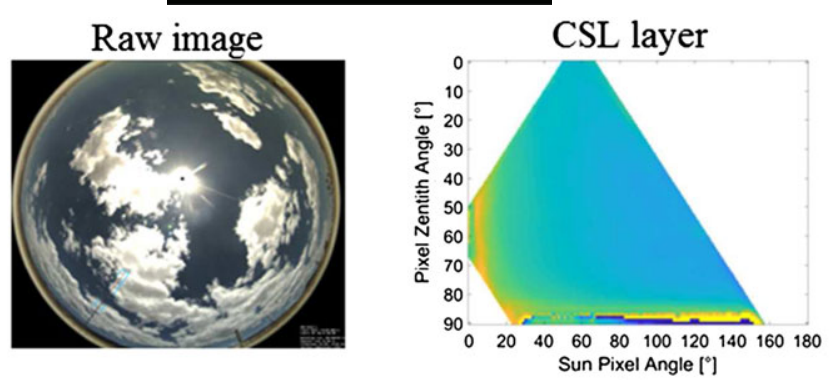

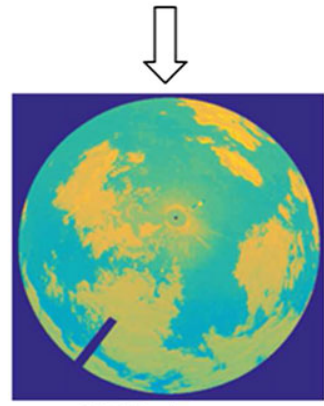

RBR image

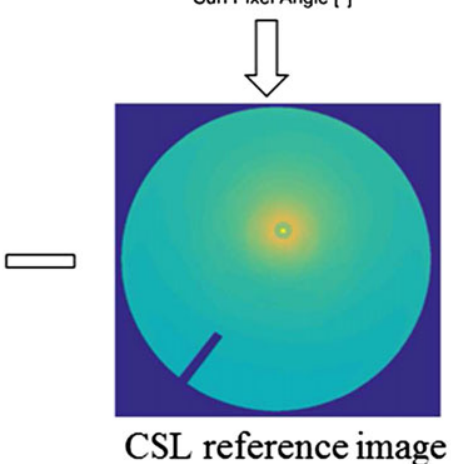

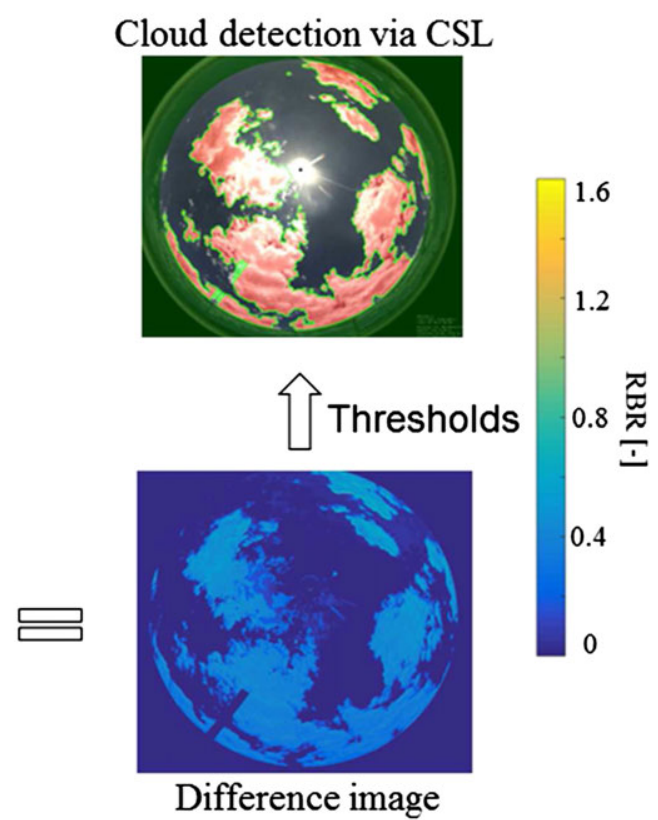

Cloud detection via CSL

1.2

$0.8 \stackrel{\text { Ш }}{\frac{\pi}{\Xi}}$

0.4

Difference image

FIGURE 4 Working principle of the clear sky library (CSL)-based cloud detection algorithm. Raw images are compared to clear sky reference images corresponding to similar Linke turbidities and air masses. One layer of the CSL is shown, showing the ratio of the corresponding red and blue CSL layers. The color bar, depicting the red-to-blue ratios (RBRs), relates to the RBR image, the CSL layer, the CSL reference image, and the difference image [Colour figure can be viewed at wileyonlinelibrary.com]
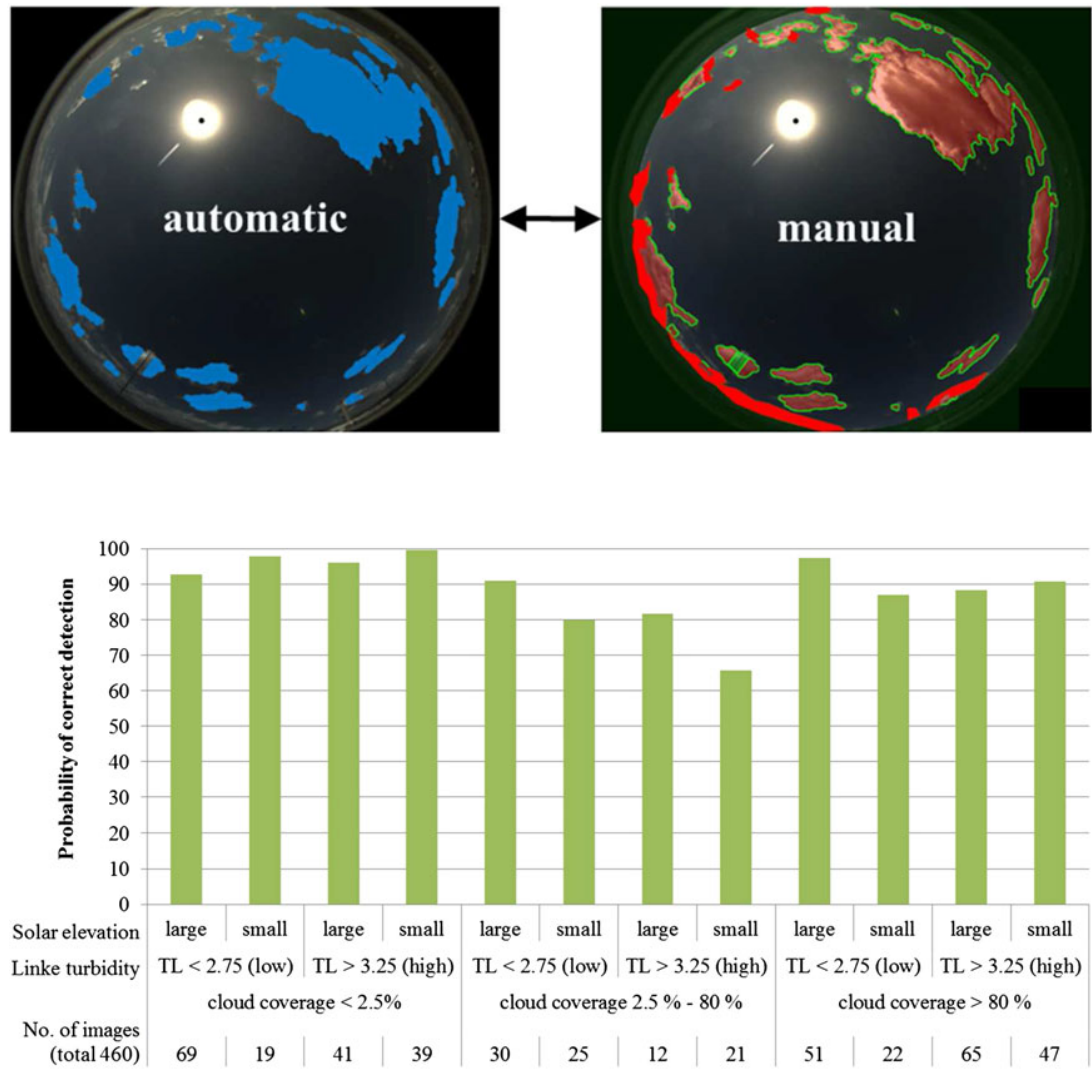

FIGURE 5 Validation approach of the clear sky library-based cloud detection algorithm: All-sky images segmented by the algorithm ("automatic," left) are compared to approximately 600 manually segmented images ("manual," right) per camera model. In this example, some clouds at the very edge of the fisheye projection are not correctly detected by the algorithm (filled red area in the left image) [Colour figure can be viewed at wileyonlinelibrary.com]

FIGURE 6 Validation results is visualized for several weather situations including large (above $30^{\circ}$ ) and small (below $20^{\circ}$ ) sun elevation angles, dusty and clear air as well as different cloud coverages [Colour figure can be viewed at wileyonlinelibrary.com] threshold-based approaches are known to struggle with overcast situations. Cloud segmentation is easiest for single-layer scattered optically thick cumulus clouds, low turbidities, and high solar elevations because of high contrast. Optically thin clouds are challenging for all segmentation approaches. Thus, the reference images for scattered cloud coverages put emphasis on such (ice) clouds. The Linke turbidity is divided into "low" with TL $<2.75$ and "high" with TL $>3.25$ based on a study of the Linke turbidity distribution as measured for our site. ${ }^{25}$

With one exception, the probability of correct detection is at $80 \%$ or above, even exceeding $95 \%$ for many situations. The probability of 
correct detection is defined as the percentage of pixels correctly detected as "cloud" or as "clear sky." This probability of correct detection drops to $66 \%$ for low solar elevations, high Linke turbidities, and medium cloud coverages between $2.5 \%$ and $80 \%$. Because of the low solar elevation and the high Linke turbidity, the saturation of the blue color of the sky is low, and the sky in these circumstances appears whitish-gray. These situations are challenging, even for a human observer, as only partially illuminated clouds do not pose a strong contrast against the aerosol layers around them and can hardly be distinguished from them. The thin border between cloud and aerosols is discussed in Calbó et al. ${ }^{27}$

For low sun elevations, the most relevant clouds near the sun are at the edge of the fisheye lens, therefore, strongly distorted and are imaged from similar angles from all 4 cameras of the WobaS-4cam system. Deriving 3-D shapes of such clouds is challenging, and small pixel-wise errors in the cloud detection result in large deviations in the corresponding 3-D positions. Thus, for sun elevations below $15^{\circ}$, a persistence forecast based on recently measured irradiance data was found to be more effective. In the following validations, timestamps with such low solar elevations are excluded. Because of the rather low irradiance levels in these situations, this approach is considered acceptable, at least for a preliminary step. If low elevations were of special interest, an additional camera tracking the sun could be used.

\section{3 | VALIDATION OF NOWCASTED IRRADIANCE MAPS}

In this section, we focus on the validation of DNI and GHI maps. These irradiance maps are the final output of the WobaS system. From DNI and GHI maps, GTI maps can be derived using regional-specific models. ${ }^{28-30}$ In Section 3.1, the irradiance maps are validated using 3 independent ground measurement stations on 30 days. These days are selected to reflect a wide range of irradiance variabilities and cloud heights. GHI, DNI, and measured cloud heights of the 30-day validation period are depicted in Figure 7.

A shadow camera system, ${ }^{31}$ providing reference irradiance maps, is used for the validation in Section 3.2. An auto-evaluation of the WobaS system was conducted on 30 days and is briefly discussed in Section 4. The validation setup at PSA is depicted in Figure 8. The 30 days of the validation are

- in 2015: September 8 to $11,15,18,19$, October 2, 4, 8 to 11,15 , 17, 18, November 14, 22, 24 to 29; and

- in 2016: May 11, 14, June 3, September 27, 28 and October 28.

As discussed in the previous section, only timestamps with minimum solar elevations of $15^{\circ}$ are considered in the validations.

Bias, relative bias, mean absolute error (MAE), relative MAE, root mean square error (RMSE), relative RMSE, standard deviation (std), and relative std are calculated for each station (Section 3.1) or each field size (Section 3.2) and each day. The relative deviations are derived from the absolute deviations and the mean irradiance measured over the validation period. The formulas are defined in the following with $o_{i}$ as the irradiance values from the observations in the reference data set and $p_{\mathrm{i}}$ as the predicted irradiance values from the nowcasted data set at timestamp index i. $N$ is the total number of timestamps included in the evaluated validation interval (excluding low elevation angles). $O_{m}$ is set to the mean observed irradiance of the validation period.

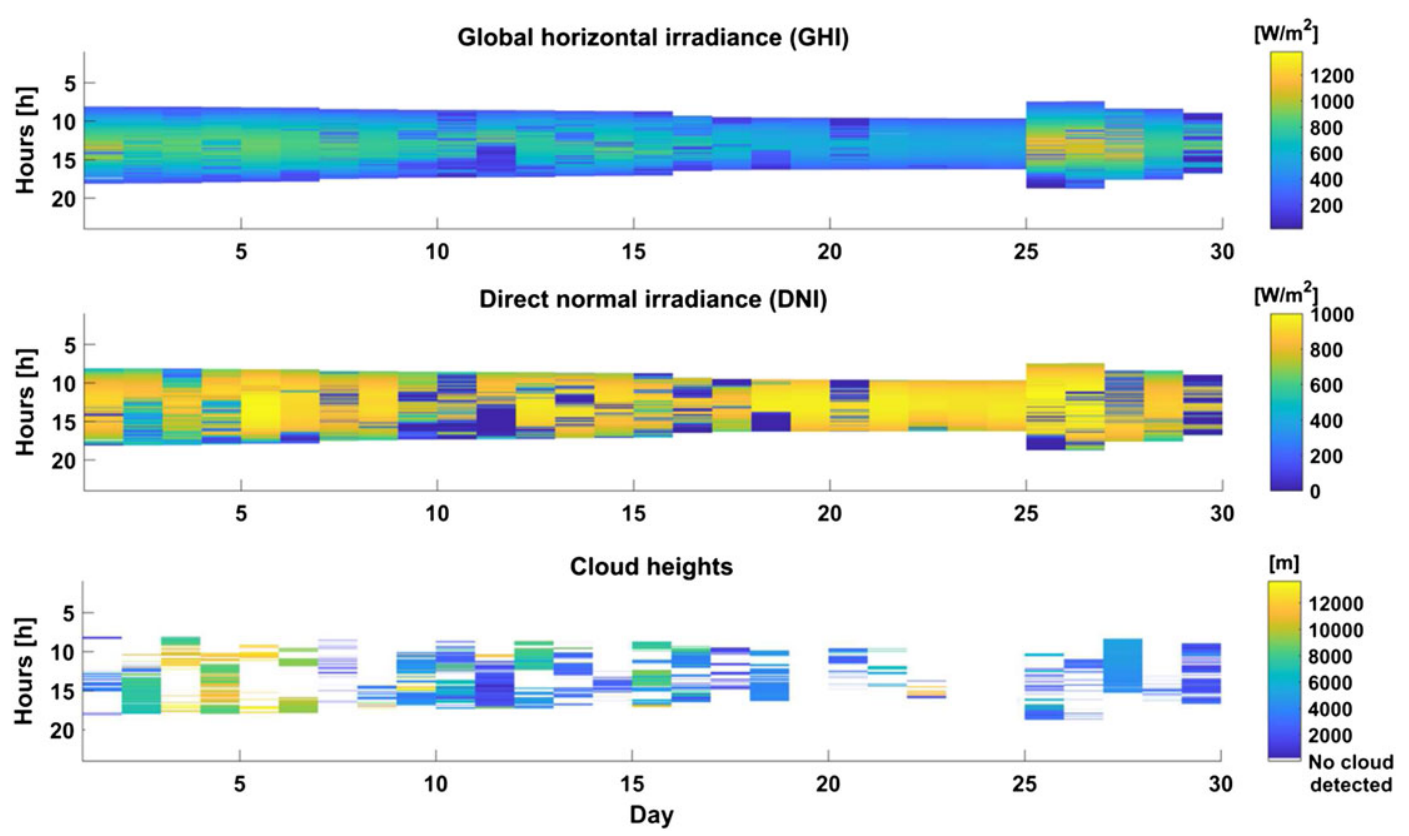

FIGURE 7 Overview of the 30-day validation period. During the validation period, a wide range of irradiance fluctuations and cloud heights (as measured by a ceilometer) are present. The pyranometer and the pyrheliometer, which measured the irradiances depicted here, are located at the southernmost $X$ in Figure 8. The ceilometer for the cloud height measurements is at the position of the white triangle in Figure 8 [Colour figure can be viewed at wileyonlinelibrary.com] 


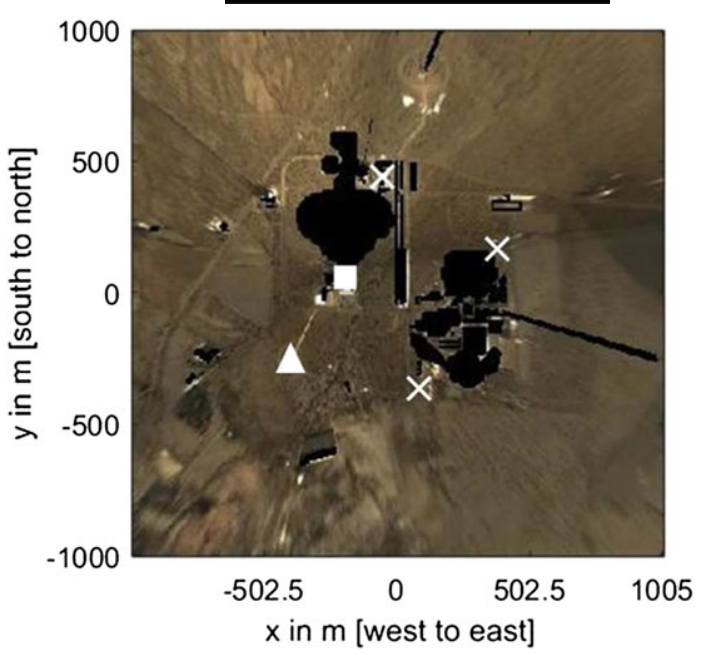

FIGURE 8 Orthoimage generated from the shadow camera system with ground measurements stations included: The square marks the position of the shadow camera system. The Xs mark the location of 3 groups comprising each a pyranometer, a pyrheliometer, and an all-sky imager. The fourth all-sky imager and a ceilometer are located at the position of the triangle [Colour figure can be viewed at wileyonlinelibrary.com]

The bias is the arithmetic average of the values of the differences:

$$
\text { bias }=\frac{1}{N} \sum_{i=1}^{N} p_{i}-o_{i}
$$

The relative bias is given by

$$
\text { relative bias }=\frac{100 \%}{O_{m}} \text { bias }
$$

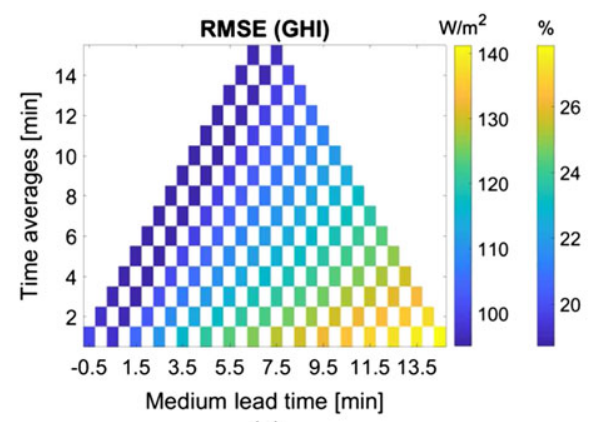

(a)

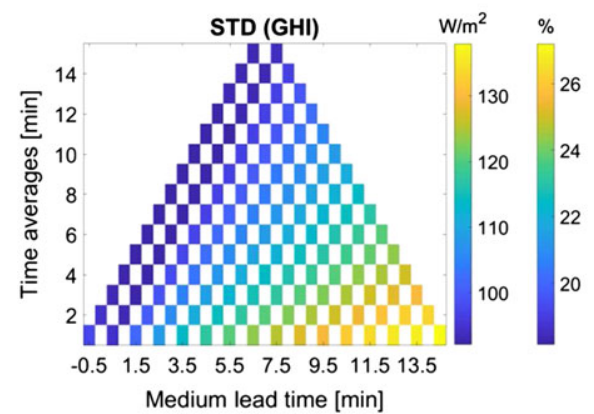

(c)
The MAE is

$$
M A E=\frac{1}{N} \sum_{i=1}^{N}\left|p_{i}-o_{i}\right|
$$

The relative MAE is defined as follows:

$$
\text { relative } M A E=\frac{100 \%}{O_{m}} M A E
$$

The RMSE is

$$
R M S E=\left[\sum_{i=1}^{N}\left(p_{i}-o_{i}\right)^{2} / N\right]^{1 / 2}
$$

The relative RMSE is defined as follows:

$$
\text { relative } R M S E=\frac{100 \%}{O_{m}} R M S E
$$

The standard deviation (std) of the fluctuations is

$$
s t d=\left[\frac{1}{N-1} \sum_{i=1}^{N}\left(\left(p_{i}-o_{i}\right)-\text { bias }\right)^{2}\right]^{1 / 2}
$$

The relative std is defined as follows:

$$
\text { relative } s t d=\frac{100 \%}{O_{m}} s t d
$$

\section{1 | Comparing nowcasts with ground measurements stations}

We look first at the root mean squared error (RMSE) and the MAE derived from 3 ground measurements stations in comparison to the

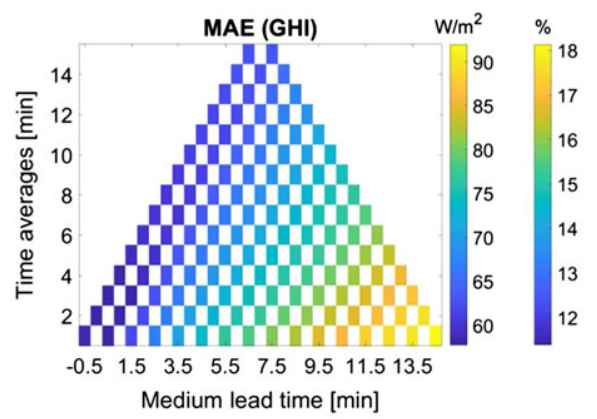

(b)

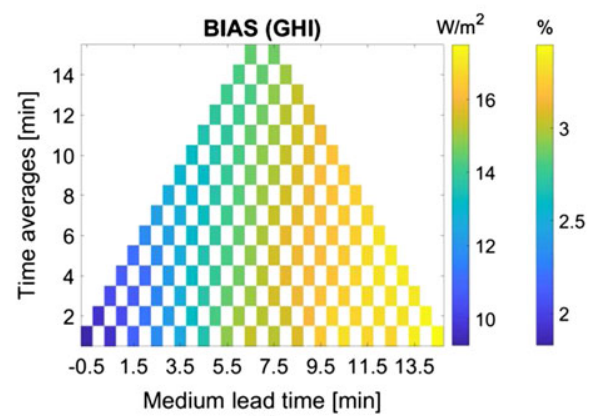

(d)

FIGURE 9 A, Absolute and relative RMSE (GHI) for a validation period of 30 days and 3 pyranometers. (B) mean absolute error (MAE), (C) standard deviation, and (D) bias. The deviations rise with higher lead times and decrease if temporal averages are considered. RMSE, root mean square error; $\mathrm{GHI}$, global horizontal irradiance [Colour figure can be viewed at wileyonlinelibrary.com] 
FIGURE 10 Field of view of a camera for cloud heights of (A) $2 \mathrm{~km}$ and (B) $10 \mathrm{~km}$. Depicted is the undistorted orthoimage derived from the fisheye projection of one camera, which highlights the occlusion from near-by objects [Colour figure can be viewed at wileyonlinelibrary.com]
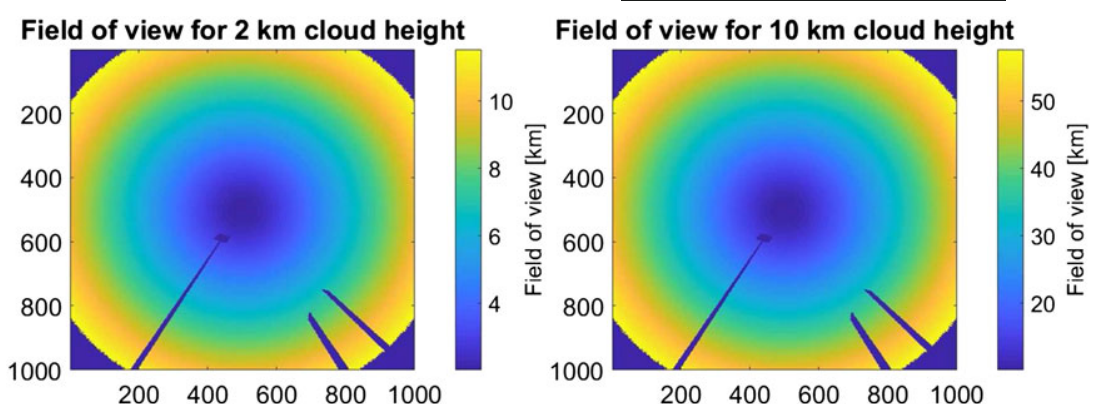

corresponding pixels in the nowcasted irradiance maps of the WobaS system over the 30 days of validation.

The deviations are depicted for $\mathrm{GHI}$ and DNI in Figures 9 and 11 , respectively. The absolute values are the average of the 90 daily stations-wise deviations (30 days and 3 stations). Each relative value is derived from the corresponding absolute values and the mean irradiance during the 30 days. On the $y$-axis, temporal averages from 1 to 15 minutes are shown. The $x$-axis shows medium lead times from -0.5 to 14.5 minutes.

Looking at Figure 9A, both the absolute and the relative RMSE values for $\mathrm{GHI}$ are displayed. As expected from the previous studies, ${ }^{32,33}$ higher lead times show higher deviations for RMSE, $\mathrm{MAE}$, and standard deviation as the more distant future is more difficult to predict. Temporal aggregations reduce these deviations because of averaging effects. However, the behavior of the bias must be explained differently: Similar to the other deviations depicted in Figure 9 , but on a lower level, the bias rises with increasing lead times and falls for higher temporal averages. Also, the bias found on 30 days is always positive (see Figure 9D).

All 3 effects hold for the depicted bias of 30 days. However, bias values for singular days within the 30 days show negative values and different behaviors. In general, there seems to be a minor tendency for positive biases within nowcasting systems (also observed in Kuhn et $\mathrm{al}^{32}$ ) as usually more clouds remain undetected than sky clear pixels being incorrectly assigned to be clouded. This is especially true for large lead times where the relevant clouds might be outside the cameras' field of view or outside the WobaS-4cam voxel space of $27.6 \mathrm{~km}^{*} 27.6 \mathrm{~km} * 10 \mathrm{~km}$. Figure 10 depicts the field of view of a camera for cloud heights of 2 and $10 \mathrm{~km}$. Clouds at an altitude of $2 \mathrm{~km}$, which are $10 \mathrm{~km}$ away can be seen in the all-sky imager. However, with cloud speeds of, eg, $20 \mathrm{~m} / \mathrm{s}$, only physical forecasts up to a lead time of 500 seconds ( $8.3 \mathrm{~min}$ ) are possible. For southern Spain, a mean cloud speed of $7.36 \mathrm{~m} / \mathrm{s}$ was found, ${ }^{19}$ resulting in an average physical forecast horizon for clouds at $2 \mathrm{~km}$ height of more than 22 minutes.
One potential reason for the underdetection of clouds and the subsequent positive bias is that the WobaS-4cam system uses its redundancy of 4 all-sky imagers to counter incorrect cloud detections: Only clouds seen by at least 3 of the 4 cameras are considered, the others are rejected. This effect potentially increases the bias, but mainly increases the robustness of the system against a camera occluded by near-by objects or dirt. With the bias ranging between $1.8 \%$ and $3.4 \%$, these effects seem to be minor compared to the standard deviation and the RMSE. In Table 1, the minimum and maximum values are shown for the error metrics as the average daily deviations of 30 days.

Figure 11 displays the error metrics found for the DNI predictions of the WobaS system similar to Figure 9. In general, the observed deviations for DNI are larger than the deviations found for the $\mathrm{GHI}$ predictions. This effect is caused by the $\mathrm{DHI}$, which damps abrupt changes by scattering irradiance onto the areas shaded by clouds. Usually, inside the shadows of cumulus clouds, the DNI drops to zero and the $\mathrm{GHI}$ is equal to the $\mathrm{DHI}$. Therefore, the relative changes of the $\mathrm{DNI}$ are larger than the relative changes of the $\mathrm{GHI}$, which results in larger deviations for the same forecasts. Besides that, the behavior of the DNI deviations is similar to the GHI deviations: Temporal aggregations reduce deviations, whereas higher lead times result in larger deviations. Table 2 shows the maximum and minimum values of the DNI deviations.

In Figure 12, temporal aggregation effects are shown differently by considering the deviations of the WobaS system for the next minutes ahead. For this so-called operator mode, the irradiances predicted for the positions of the ground measurements stations and the average of the next $\mathrm{X}$ min are compared to the aggregated measurements of these stations. The error metrics for $\mathrm{GHI}$ and $\mathrm{DNI}$ are depicted respectively in Figure 12A,B. Because of temporal aggregations, the predictions for the next minute and the temporally aggregated predictions for the next 15 minutes ahead show similar deviations. These deviations are of interest if a back-up electricity generator of a solar plant requires time to be operational and the aggregated irradiance for the next, eg, 15 minutes

TABLE 1 Minimum and maximum GHI deviations found as the average daily and station-wise deviations on $30 \mathrm{~d}$ (compare with the graphs in Figure 9)

\begin{tabular}{lllllll} 
GHI Metrics & Minimum Value & $\begin{array}{l}\text { Medium Lead Time, } \\
\min \end{array}$ & $\begin{array}{l}\text { Temporal Average, } \\
\min \end{array}$ & Maximum Value & $\begin{array}{l}\text { Medium Lead Time, } \\
\min \end{array}$ & $\begin{array}{l}\text { Temporal Average, } \\
\text { min }\end{array}$ \\
\hline RMSE & $18.7 \%\left(95 \mathrm{~W} / \mathrm{m}^{2}\right)$ & 2.5 & 7 & $27.9 \%\left(141 \mathrm{~W} / \mathrm{m}^{2}\right)$ & 14.5 & 1 \\
MAE & $11.4 \%\left(58 \mathrm{~W} / \mathrm{m}^{2}\right)$ & 1 & 4 & $18.1 \%\left(92 \mathrm{~W} / \mathrm{m}^{2}\right)$ & 14.5 & 1 \\
Standard deviation & $18.2 \%\left(92 \mathrm{~W} / \mathrm{m}^{2}\right)$ & 2.5 & 7 & $27.2 \%\left(138 \mathrm{~W} / \mathrm{m}^{2}\right)$ & 14.5 & 1 \\
Bias & $1.8 \%\left(9 \mathrm{~W} / \mathrm{m}^{2}\right)$ & -0.5 & 1 & $3.4 \%\left(17 \mathrm{~W} / \mathrm{m}^{2}\right)$ & 14.5 & 1 \\
\hline
\end{tabular}

Abbreviations: GHI, global horizontal irradiance; MAE, mean absolute error; RMSE, root mean square error. 


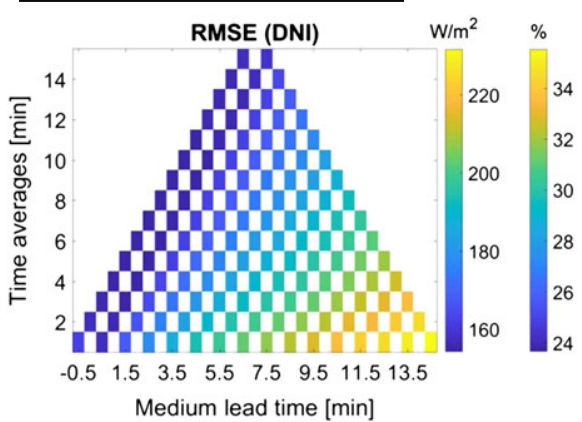

(a)

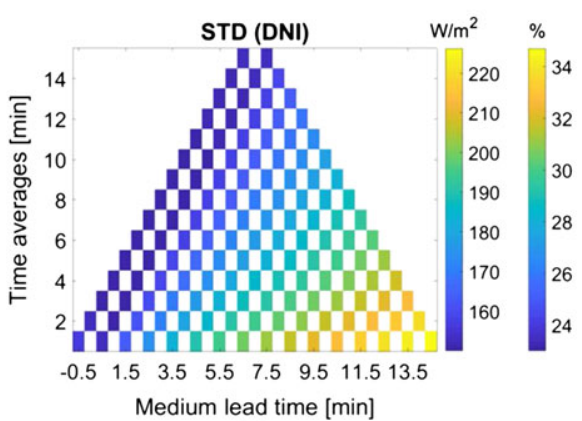

(c)

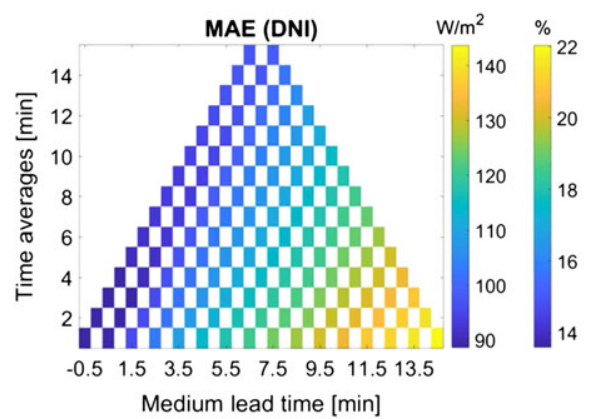

(b)

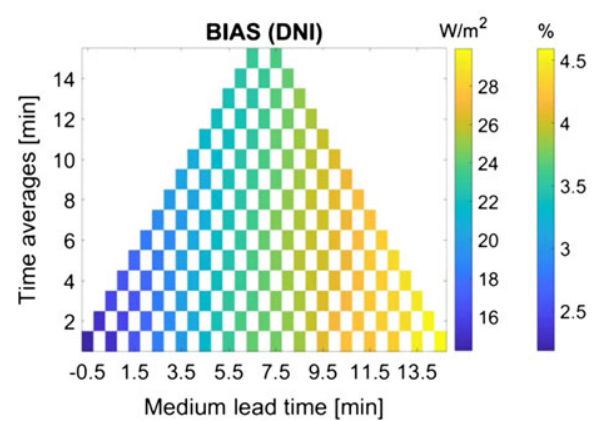

(d)

FIGURE 11 (A) Absolute and relative RMSE (DNI) for a validation period of 30 days and 3 pyrheliometers. (B) mean absolute error (MAE), (C) standard deviation, (D) bias. The deviations rise with higher lead times and decrease if temporal averages are considered. RMSE, root mean square error; DNI, direct normal irradiance [Colour figure can be viewed at wileyonlinelibrary.com]

TABLE 2 Minimum and maximum DNI deviations found as the average daily and station-wise deviations on $30 \mathrm{~d}$ (compare with the graphs in Figure 11)

\begin{tabular}{lllllll} 
DNI Metrics & Minimum Value & $\begin{array}{l}\text { Medium Lead Time, } \\
\min \end{array}$ & $\begin{array}{l}\text { Temporal Average, } \\
\min \end{array}$ & $\begin{array}{l}\text { Maximum Value, } \\
\min \end{array}$ & $\begin{array}{l}\text { Medium Lead Time, } \\
\min \end{array}$ & $\begin{array}{l}\text { Temporal Average, } \\
\text { min }\end{array}$ \\
\hline RMSE & $23.7 \%\left(154 \mathrm{~W} / \mathrm{m}^{2}\right)$ & 2.5 & 7 & $35.5 \%\left(232 \mathrm{~W} / \mathrm{m}^{2}\right)$ & 14.5 & 1 \\
MAE & $13.6 \%\left(89 \mathrm{~W} / \mathrm{m}^{2}\right)$ & 0.5 & 3 & $22.0 \%\left(144 \mathrm{~W} / \mathrm{m}^{2}\right)$ & 14.5 & 1 \\
Standard deviation & $23.0 \%\left(150 \mathrm{~W} / \mathrm{m}^{2}\right)$ & 2.5 & 7 & $34.7 \%\left(226 \mathrm{~W} / \mathrm{m}^{2}\right)$ & 14.5 & 1 \\
Bias & $2.2 \%\left(14 \mathrm{~W} / \mathrm{m}^{2}\right)$ & -0.5 & 1 & $4.6 \%\left(30 \mathrm{~W} / \mathrm{m}^{2}\right)$ & 14.5 & 1 \\
\hline
\end{tabular}

Abbreviations: DNI, direct normal irradiance; MAE, mean absolute error; RMSE, root mean square error.

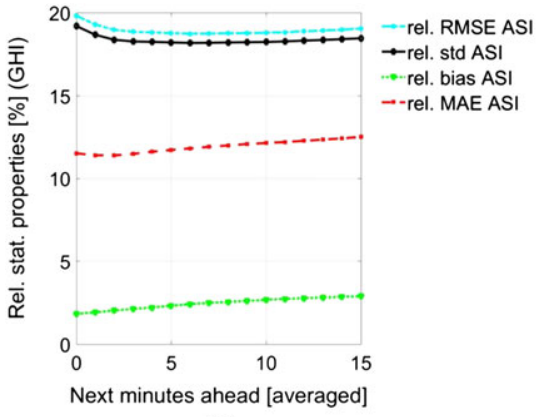

(a)

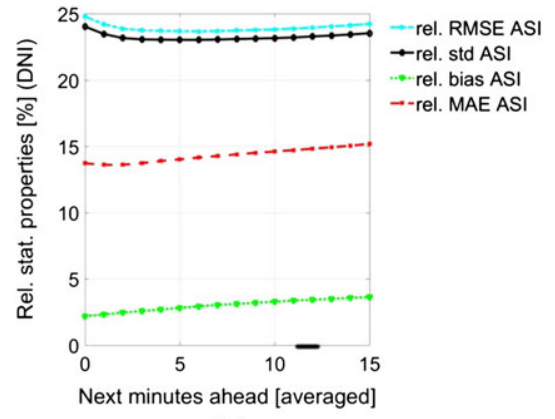

(b)
FIGURE 12 Error metrics for global horizontal irradiance $(\mathrm{GHI})(\mathrm{A})$ and direct normal irradiance (DNI) (B) using the operator mode. Three ground-based reference stations are compared to corresponding pixels in the nowcasted irradiance maps for temporal averages up to $15 \mathrm{~min}$. Depicted are the relative deviations of 30 days. Because of temporal averaging, the deviations for the next minute ahead and the deviations for the next 15 min ahead are found to be similar. This effect is of interest for plants with back-up generators. ASI, all-sky imager; RMSE, root mean square error; MAE, mean absolute error [Colour figure can be viewed at wileyonlinelibrary.com] must be predicted. The deviations displayed in Figure 12 correspond to the left-most value of each row in each graph of Figures 9 and 11. Note the difference in the $x$-axis in these graphs: In Figures 9 and 11, the medium lead times for various temporal averages are considered, whereas in Figure 12 the deviations for the next minutes ahead are displayed. 
In the graphs of Figure 12 ("ASI"-all-sky imager-based WobaS system), the RMSE and MAE deviations found for 0 minute ahead are slightly higher than the deviations found for 15 minutes ahead (RMSE [GHI]: 19.8\% [0 $\mathrm{min}$ ] to $19.0 \%$ [15 min]; RMSE [DNI]: $24.8 \%$ [0 $\mathrm{min}$ ] to $24.2 \%$ [15 $\mathrm{min}$ ]). This is caused by interplay of 2 effects: With larger lead times, the predictions become less accurate, which can be seen in the graphs of Figures 9 and 11. On the other hand, it is also visible in these graphs that with larger temporal aggregations, the deviations are reduced. In the operator modus, these 2 effects are combined and balance themselves: The increase of the deviations with larger lead times is compensated by the larger temporal aggregations.

The results found for the WobaS system are in alignment with publications on other all-sky imager-based nowcasting systems: RMSE values between 155 and $250 \mathrm{~W} / \mathrm{m}^{2}$ for 10 minutes GHI forecasts are reported in Bernecker et $\mathrm{al}^{34}$ (validated on $15 \mathrm{~d}$ in Germany) and RMSE values for $\mathrm{GHI}$ forecasts up to $250 \mathrm{~W} / \mathrm{m}^{2}$ for 23 minutes lead time in Schmidt et $\mathrm{al}^{35}$ for a validation period of 2 months in Germany. A two all-sky imager-based system validated on 9 days at the same location as the WobaS system (Spain) showed larger deviations for 1 minute averages of above $250 \mathrm{~W} / \mathrm{m}^{2}$ (RMSE, DNI) and similar deviations (RMSE, DNI: 31.6\%, lead time $15 \mathrm{~min}$ ) for the operator mode. ${ }^{32} \mathrm{~A}$ hybrid all-sky imager/stochastic learning approach presented in Xia et al ${ }^{21}$ achieved RMSE values between 55 and $140 \mathrm{~W} / \mathrm{m}^{2}$ for DNI forecasts and a lead time of 10 minutes on a validation period of 6 months in California, United States. In Fu et al, ${ }^{36}$ RMSE values for DNI forecasts between $169 \mathrm{~W} / \mathrm{m}^{2}$ (25\%) for 5 minutes lead time and $191 \mathrm{~W} / \mathrm{m}^{2}$ (28\%) for 15 minutes lead time as well as MAE values between $139 \mathrm{~W} / \mathrm{m}^{2}$ (22\%, $\left.5 \mathrm{~min}\right)$ and $152 \mathrm{~W} / \mathrm{m}^{2}(24 \%, 15 \mathrm{~min})$ are found on a 4-week validation period in Taiwan.
Comparing observed deviations of nowcasting systems, which were validated on different days and in different weather situations, must be done with extreme precaution: The specific weather situations strongly influence the performance of nowcasting systems and the days included in the validation have a strong impact on the final error metrics. For the 30-day validation period used for the WobaS system, a wide range of different weather situations was chosen (Figure 7). Moreover, the weather above southern Spain with multiple cloud layers being frequently present might be more complex than the weather elsewhere. For instance, on the pacific coast of the United States, cumulus clouds at low altitudes seem to be predominantly present. ${ }^{37}$

\subsection{Comparing nowcasts with a shadow camera-based reference system}

At the Plataforma Solar de Almería, Spain, a unique and innovative shadow camera system is operational, ${ }^{31}$ which provides reference irradiance maps for the validation of nowcasts. ${ }^{10,32}$ The shadow camera system consists of 6 cameras taking photos from the top of an 87-m high tower. Out of the 6 photos, taken every 15 seconds, an orthoimage is calculated. To detect shadows in the current orthoimage, 2 reference orthoimages corresponding to similar solar positions are compared to the current orthoimage. One reference orthoimage was taken when no shadow fell on the imaged area. The second orthoimage was taken when the whole imaged area was shaded. Using the 2 reference orthoimages, consisting out of 6 raw images each, the current orthoimage can be segmented into shaded and unshaded areas. For the irradiances in the unshaded area, the clear sky irradiance is taken. The irradiances in the shaded areas are derived from the camera measurements, which are normalized with high-
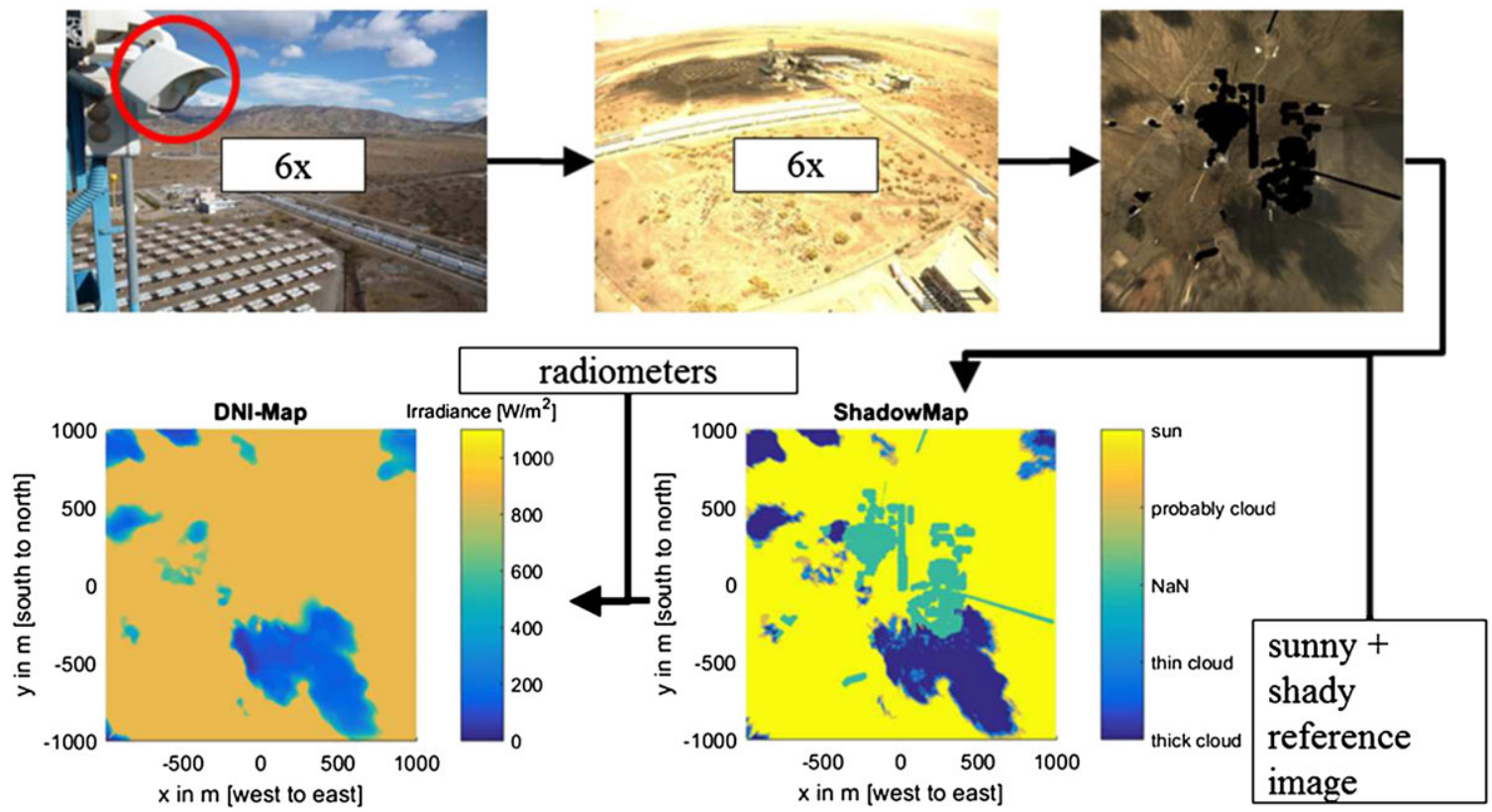

FIGURE 13 Working principle of the shadow camera system, providing reference irradiance maps for the WobaS system. Six downward-facing cameras acquire an image every $15 \mathrm{~s}$. The set of 6 images are combined to an orthoimage. With reference orthoimages, taken during cloud-free and overcast conditions, available, shadows are detected and irradiance maps in high spatial $(25 \mathrm{~m} 2)$ and temporal resolutions of $15 \mathrm{~s}$ are generated. DNI, direct normal irradiance [Colour figure can be viewed at wileyonlinelibrary.com] 
precision irradiance measurements. Thus, irradiance maps (DNI, GHI, GTI) for the current situation are calculated. These irradiance maps have a spatial resolution of $25 \mathrm{~m}^{2}$ over an area of $4 \mathrm{~km}^{2}$. Comparing pixels of the derived irradiance maps to corresponding ground measurements, the shadow camera system shows RMSE (DNI) between $4.2 \%$ and $16.7 \%$ for 1 minute averages. RMSE (GHI) deviations are below $10 \%$. Like the WobaS system, the shadow camera system benefits from spatial and temporal averaging with resulting spatially aggregated RMSE (GHI) between $3.3 \%$ and $8.7 \%$ and RMSE (DNI) between $4.3 \%$ and $10 \%$. Figure 13 illustrates the approach. The shadow camera system is presented in detail in Kuhn et al. ${ }^{31}$ Using the spatially resolved irradiance maps generated by the shadow camera system, spatial aggregation effects regarding the deviations

19.09.2015:

LT $15 \mathrm{~min}$, AVG $1 \mathrm{~min}$
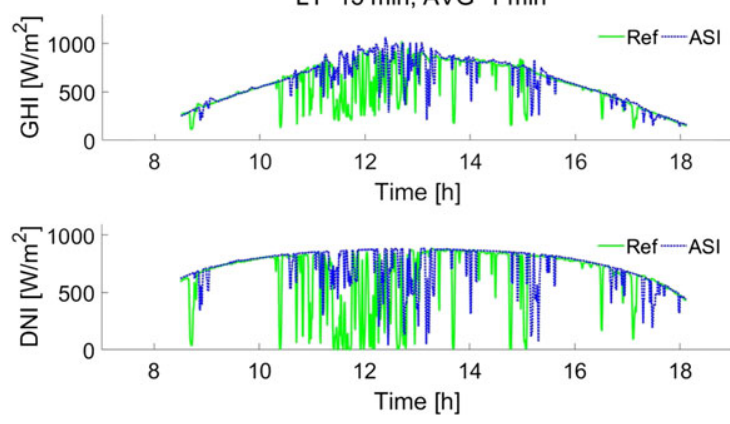

FIGURE 14 Global horizontal irradiance (GHI) and direct normal irradiance (DNI) ground measurements (green, "Ref"), compared to the WobaS forecasts of the pixel corresponding to the measurement station (blue, "ASI"-all-sky imager) for lead time 15 min ("LT") and 1 min time average ("AVG"). As can be seen, this example day shows a high variability [Colour figure can be viewed at wileyonlinelibrary.com] of nowcasting systems can be studied. Studying spatial aggregation effects on forecasting deviations is of importance as these effects are inherently present in industrial solar power plants, covering up to several square kilometers.

The effects of spatial aggregations are discussed by looking at one example day (September 9, 2015). The irradiances of this day are depicted in Figure 14, showing a high level of variability.

Figure 15 displays the $\mathrm{GHI}$ deviations found for the WobaS system in comparison to the shadow camera system for 1-minute temporal averages. Considered field sizes vary between $25 \mathrm{~m}^{2}$ and $4 \mathrm{~km}^{2}$ ( $x$-axis). Lead times from 0 to 15 minutes are depicted on the $y$-axis. The deviations increase with larger lead times. Because of spatial aggregation effects, the deviations decrease for larger field sizes. For instance, an RMSE value of $21.4 \%\left(120 \mathrm{~W} / \mathrm{m}^{2}\right)$ is found for lead time 0 minute and a field size of $25 \mathrm{~m}^{2}$, which shrinks to $13.0 \%$ (73 W/ $\mathrm{m}^{2}$ ) for a field size of $4 \mathrm{~km}^{2}$.

The DNI deviations are depicted in Figure 16. Because of effects explained in the previous section, the deviations observed for the $\mathrm{DNI}$ are larger than the GHI deviations. In general, the DNI deviations show similar behavior in comparison to the GHI deviations. However, the DNI deviations drop more for larger field sizes. This effect can be explained with typical cloud sizes of this day: If clouds on that day have a usual diameter of 500 to $1000 \mathrm{~m}$, then for field sizes above $1000 \mathrm{~m}$ the exact position of the cloud inside the considered field is not relevant for spatially aggregated irradiances. Prior to this critical size, small errors in the predictions of the shadow locations lead to large deviations as the shadow misses the field completely. This drop in the deviations is blurred out for the $\mathrm{GHI}$ because of the $\mathrm{DHI}$ reducing the offsets for small field sizes.

In the previous section, the operator mode was introduced. The operator mode considers deviations for the next minutes ahead.

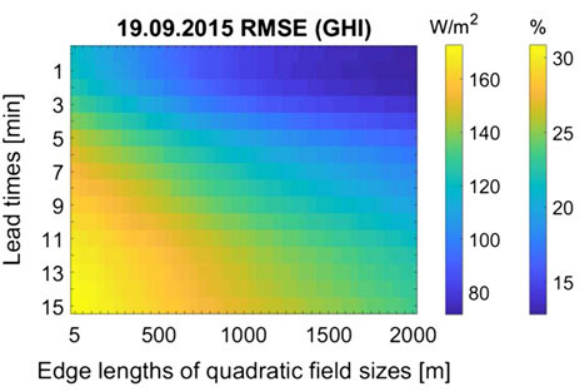

(a)

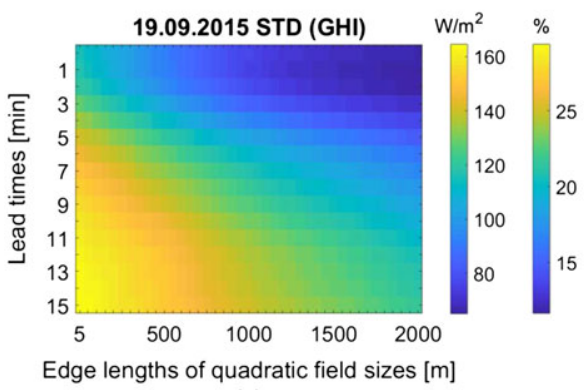

(c)

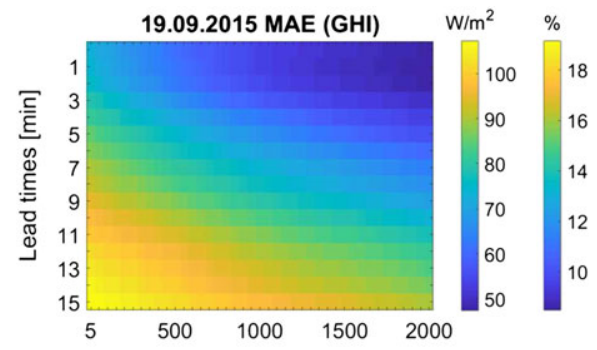

Edge lengths of quadratic field sizes [m]

(b)

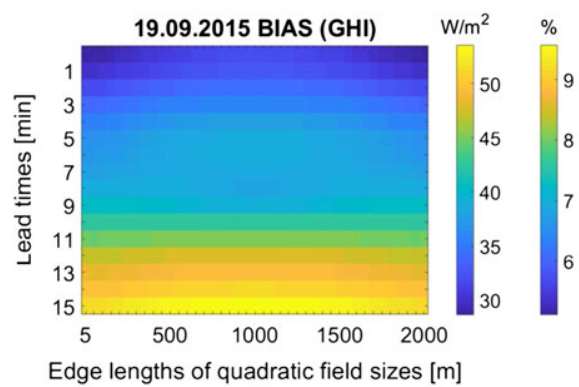

(d)

FIGURE 15 Absolute and relative error metrics for the 1-min global horizontal irradiance (GHI) forecasted by the WobaS system in comparison to the shadow camera system for aggregated field sizes from $25 \mathrm{~m} 2$ to $4 \mathrm{~km} 2$ and lead times ranging from 0 to 15 min. MAE, mean absolute error [Colour figure can be viewed at wileyonlinelibrary.com] 


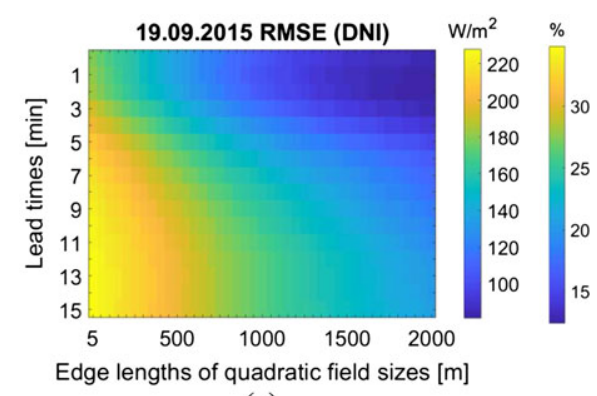

(a)

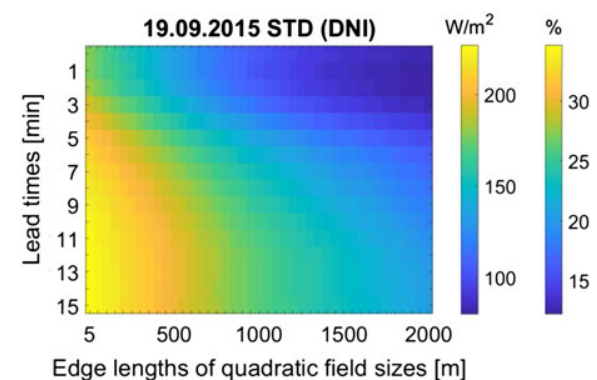

(c)

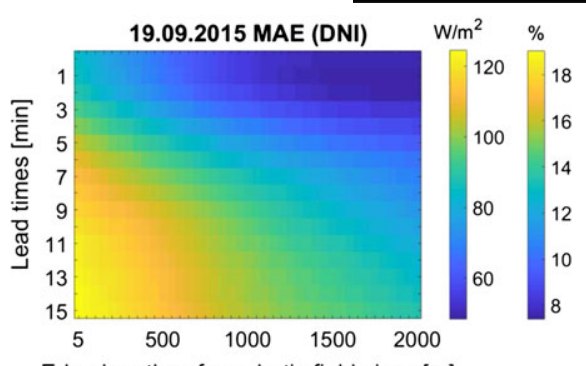

Edge lengths of quadratic field sizes [m]

(b)

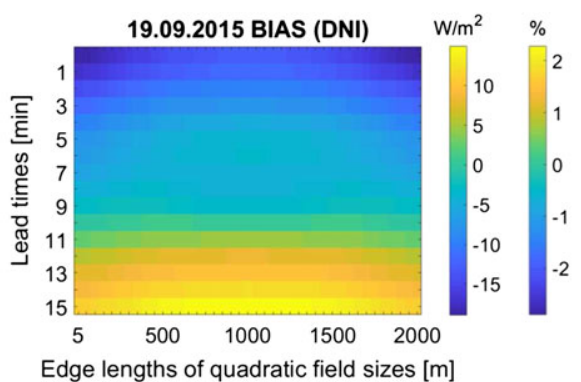

(d)

FIGURE 16 Direct normal irradiance (DNI) deviations corresponding to Figure 15. MAE, mean absolute error [Colour figure can be viewed at wileyonlinelibrary.com]

Because of temporal aggregation effects, the deviations found for the next minute ahead and the next 15 minutes ahead are similar if pixels in the nowcasted irradiance maps are compared to reference ground measurements (see Figure 12 and corresponding discussion). In Figures 17 (GHI) and 18 (DNI), the operator mode is depicted considering different field sizes, lead times, and deviations.

As an example, for field sizes of $4 \mathrm{~km}^{2}$ and considering the next 4 minutes ahead, the observed relative RMSE values are $12.5 \%$ for $\mathrm{GHI}$ (Figure 17A). Without using the operator mode, the deviation observed for a lead time of 4 minutes and field sizes of $4 \mathrm{~km}^{2}$ correspond to an RMSE of 15.0\% (GHI), as shown in Figure 15A. If only 1 pixel is considered, temporal aggregation reduces the deviations from $24.9 \%$ (RMSE, GHI, lead time $4 \mathrm{~min}$ ) to $20.3 \%$ (next 4 min ahead). For DNI deviations, temporal aggregations reduce the deviations found for 1 pixel from RMSE 30.6\% (Figure 16A, lead time $4 \mathrm{~min}$ ) to RMSE 26.2\% (Figure 18A, next 4 min ahead). For 1 pixel, temporal aggregations also reduce the DNI deviations from RMSE 34.8\% (lead time $15 \mathrm{~min}$ ) to RMSE $27.6 \%$ (next $15 \mathrm{~min}$ ahead). For field sizes of

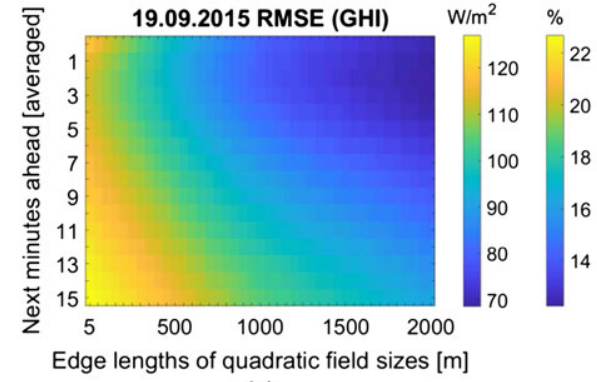

(a)

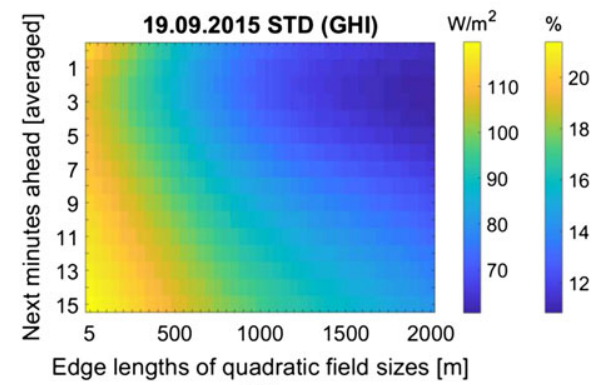

(c)

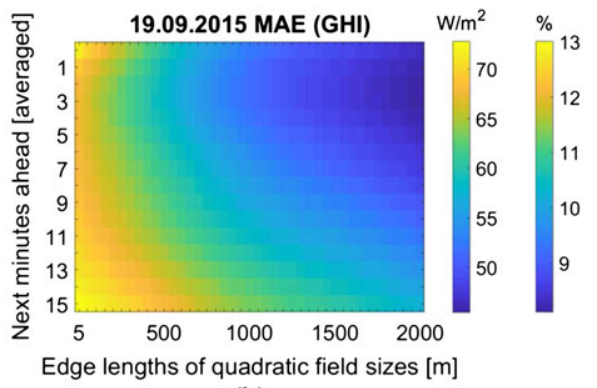

(b)

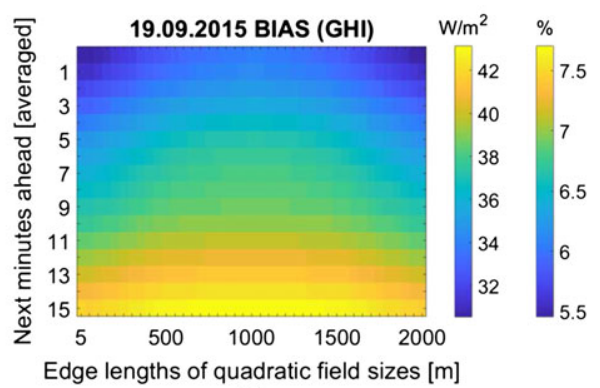

(d)

FIGURE 17 Global horizontal irradiance (GHI) deviations for the operator modus, various field sizes, and lead times, in comparison to the shadow camera system. MAE, mean absolute error [Colour figure can be viewed at wileyonlinelibrary.com] 


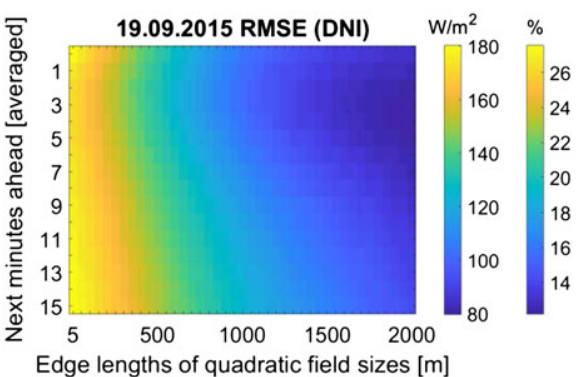

(a)

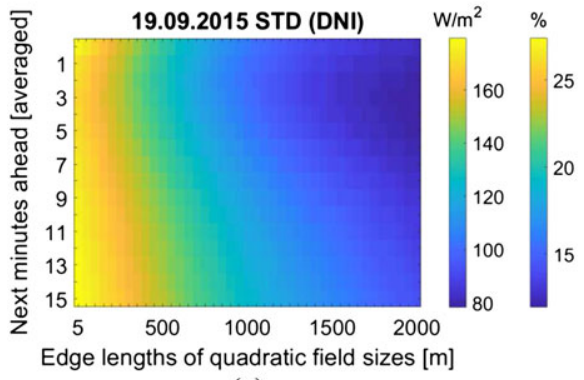

(c)

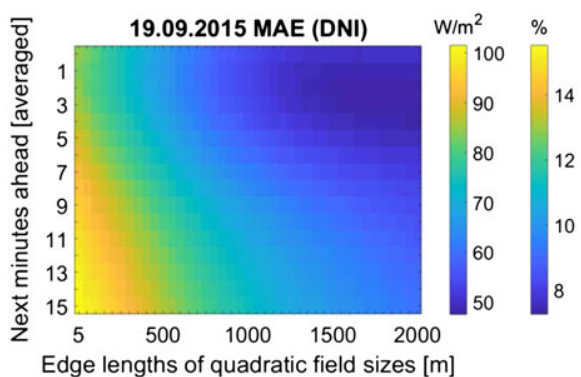

(b)

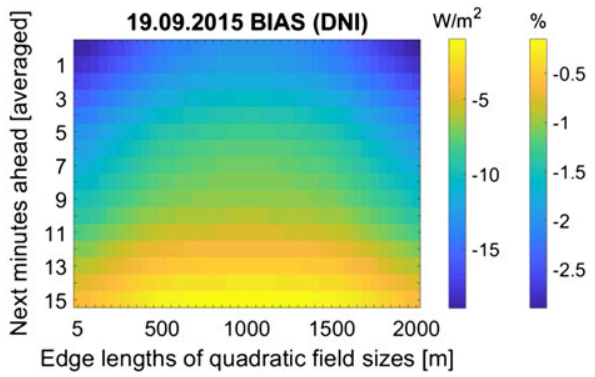

(d)

FIGURE 18 Direct normal irradiance (DNI) deviations corresponding to Figure 17. MAE, mean absolute error [Colour figure can be viewed at wileyonlinelibrary.com]

$4 \mathrm{~km}^{2}$ and considering the next 15 minutes ahead, spatio-temporal aggregation reduces the RMSE to $14.2 \%$ (Figure 18A).

As illustrated with 1 day considered as an example in this section, spatial aggregation effects significantly reduce forecasting errors. Since spatial aggregation effects are present in every industrial solar plant, these effects must be considered in the validations of nowcasting systems. For the total dispatched power of solar plants, this effect is discussed in Marcos et al. ${ }^{38}$

In the absence of a shadow camera system acting as a reference, auto-evaluations could be considered. For the whole validation period, such auto-evaluations of the WobaS-4cam system were performed. Auto-evaluations compare predicted irradiance maps for future timestamps with irradiance maps predicted on and for these timestamps. The WobaS-4cam system thus evaluates itself ("auto-evaluation"). Auto-evaluations of nowcasting systems should be treated with great precaution, especially regarding irradiance forecasts. In direct comparison, both the values of the deviations and their behavior as observed via auto-evaluations differ significantly from the deviations observed via reference measurements. Auto-evaluations could be used to test the robustness of a nowcasting system as well as the combined errors of inconsistent cloud detections, tracking errors, and deviations in cloud height determinations. However, it is suggested to validate these subtasks separately with additional references to enable optimization. Validating subtask separately leads to small improvements, which in the end add up to a generally improved and robust nowcasting system.

\section{4 | CONCLUSION AND FUTURE WORK}

In this publication, the WobaS-4cam nowcasting system was validated on 30 days representing various weather conditions with special focus on temporal and spatial aggregation effects. Temporal aggregation effects are relevant for defining storage requirements. Spatial aggregation effects are inherent in industrial-size solar plants when the resulting energy production is under consideration. The deviations observed for the WobaS-4cam system for 1-minute averages are coherent with other recently published nowcasting systems. However, directly comparing nowcasting systems using different data sets is delicate as the specifics of the meteorological situation during the selected days strongly influence the error metrics.

As key findings, both temporal and spatial aggregation significantly reduces forecasting errors. This was not studied in detail before for the temporal and spatial resolutions considered in this publication. Indeed, most nowcasting systems are validated on the basis of few ground measurement stations, which yields error metrics that do not represent fitness-for-use and might not be relevant for industrial applications. The validation period should reflect all possible weather situations and their relative occurrence. In literature, because of technical constraints, often only few days are used for validation and this could be critical for the representability of the conclusions. Spatial aggregations are found to reduce RMSE (GHI) values from $21.4 \%$ to $13.0 \%$ for lead time 0 minute and field sizes between $25 \mathrm{~m}^{2}$ and $4 \mathrm{~km}^{2}$. Temporal averaging reduces RMSE (GHI) deviations from $25.3 \%$ (medium lead time $7.5 \mathrm{~min}, 1 \mathrm{~min}$ temporal average) to $19.0 \%$ (medium lead time $7.5 \mathrm{~min}, 15 \mathrm{~min}$ temporal average). Furthermore, the effects of increased accuracies for larger temporal aggregations and increased deviations for larger lead times are found to be equivalent. Another key finding concerns auto-evaluations, which were found unfit for the validation of irradiance maps.

The WobaS-4cam system is currently operational at a commercial solar power plant. In the near future, nowcasting systems such as the WobaS systems will contribute to handle ramp rate limitations. By providing nowcasts, the WobaS-4cam system helps to comply with 
such regulations, eg, by reducing the required battery size. The conducted validation found the WobaS-4cam system to be reliable for operation in industry. Thus, with all-sky imager-based nowcasting tools and relatively small batteries, the cloud induced variability of the solar resource will not lead to severe instabilities of electrical grids.

Future work will conduct benchmarking of different 1 and 2 camera-based nowcasting systems in comparison to the WobaS4cam system presented here. Moreover, there will be an on-going optimization of all WobaS nowcasting systems. This way, the WobaS nowcasting family will be improved step by step.

\section{ACKNOWLEDGEMENTS}

The research presented in this publication has received funding from the German Federal Ministry for Economic Affairs and Energy within the WobaS project, which enabled the development of the WobaS nowcasting system and the creation of reference irradiance maps from the shadow camera system. The initial development of the shadow camera system was partially financed by the European Union's Horizon 2020 programme (PreFlexMS, Grant Agreement no. 654984). The European Union's FP7 programme partially financed operations of the all-sky imagers and other ground measurements (DNICast project, Grant Agreement no. 608623).

\section{ORCID}

Pascal Kuhn (1) http://orcid.org/0000-0001-9978-5706

\section{REFERENCES}

1. Crăciun BI, Kerekes T, Séra D, Teodorescu R, Annakkage UD. Power ramp limitation capabilities of large PV power plants with active power reserves. IEEE Trans Sustainable Energy. 2017;8(2):573-581. https://doi. org/10.1109/tste.2016.2612121

2. Gevorgian, V. and S. Booth, Review of PREPA technical requirements for interconnecting wind and solar generation, NREL/TP; 5 D 0057089, 2013, available online: http://www.nrel.gov/docs/fy14osti/ 57089.pdf

3. Chow CW, Urquhart B, Lave M, et al. Intra-hour forecasting with a total sky imager at the UC San Diego solar energy testbed. Sol Energy. 2011;85(11):2881-2893. https://doi.org/10.1016/j.solener.2011.08.025

4. Cheng, H.Y. and C.C. Yu. Solar irradiance now-casting with ramp-down event prediction via enhanced cloud detection and tracking. in 2016 IEEE International Conference on Multimedia and Expo (ICME). 2016. https://doi.org/10.1109/icme.2016.7552863.

5. Ding, Y., X. Cheng, F. Cui, X. Zhu, and H. Zhou. Very-short term forecast of global horizontal irradiance based on ground-based sky imager and lifted condensation level calculation. in 2014 China International Conference on Electricity Distribution (CICED). 2014. https://doi.org/ 10.1109/ciced.2014.6991849.

6. Yang H, Kurtz B, Nguyen D, et al. Solar irradiance forecasting using a ground-based sky imager developed at UC San Diego. Sol Energy. 2014;103:502-524. https://doi.org/10.1016/j.solener.2014.02.044

7. Najera, Y., D.R. Reed, and W.M. Grady. Image processing methods for predicting the time of cloud shadow arrivals to photovoltaic systems. In 2011 37th IEEE Photovoltaic Specialists Conference. 2011. https:// doi.org/10.1109/PVSC.2011.6185877

8. Dev, S., F.M. Savoy, Y.H. Lee, and S. Winkler. Estimation of solar irradiance using ground-based whole sky imagers. In 2016 IEEE International Geoscience and Remote Sensing Symposium (IGARSS). 2016. https:// doi.org/10.1109/igarss.2016.7730887

9. Sukič P, Štumberger G. Intra-minute cloud passing forecasting based on a low cost loT sensor-a solution for smoothing the output power of PV power plants. Sensors. 2017;17(5):1116. https://doi.org/10.3390/ s17051116

10. Blanc, P., P. Massip, A. Kazantzidis, P. Tzoumanikas, P. Kuhn, S. Wilbert, D. Schüler, and C. Prahl. Short-term forecasting of high resolution local DNI maps with multiple fish-eye cameras in stereoscopic mode. in AIP conference proceedings (SolarPACES), accepted for publication. 2016.

11. Wilbert, S., B. Nouri, P. Kuhn, D. Schüler, C. Prahl, N. Kozonek, R. PitzPaal, T. Schmidt, N. Kilius, M. Schroedter-Homscheidt, and Z. Yasser. Wolkenkamera-basierte Kürzestfristvorhersage der Direktstrahlung. in 19. Kölner Sonnenkolloquium 2016. Köln, Germany.

12. Barnes AK, Balda JC, Escobar-Mejía A. A semi-Markov model for control of energy storage in utility grids and microgrids with PV generation. IEEE Trans Sustainable Energy. 2015;6(2):546-556. https:// doi.org/10.1109/tste.2015.2393353

13. Sani Hassan A, Cipcigan L, Jenkins N. Optimal battery storage operation for PV systems with tariff incentives. Appl Energy. 2017;203:422-441. https://doi.org/10.1016/j.apenergy.2017.06.043

14. West SR, Rowe D, Sayeef S, Berry A. Short-term irradiance forecasting using skycams: motivation and development. Sol Energy. 2014;110:188-207. https://doi.org/10.1016/j.solener.2014.08.038

15. Wilbert, S. and E. Reuschenbach. Solar researchers demonstrate new solar irradiance nowcasting system in a 50-megawatts solar power plant. 2017 [cited 2017 June 30]; Available from: http://www.dlr.de/ sf/en/desktopdefault.aspx/tabid-10436/12676_read-48274/

16. Aga V, Peruchena CF. PreFlexMS: predictable flexible molten salts solar power plants. Impact. 2017;2017(3):58-60. https://doi.org/10.21820/ 23987073.2017.3.58

17. Oberländer, D., C. Prahl, S. Wilbert, S. Müller, B. Stanicki, and N. Hanrieder. Cloud shadow maps from whole sky imagers and voxel carving. in ICEM. 2015. Boulder.

18. Kuhn P, Wirtz $M$, Killius $N$, et al. Benchmarking three low-cost, low-maintenance cloud height measurement systems and ECMWF cloud heights. Sol Energy (under review). 2017;

19. Kuhn P, Wirtz M, Wilbert S, et al. Field validation and benchmarking of a cloud shadow speed sensor. Sol Energy (under review). 2017;

20. Taravat A, Frate FD, Cornaro C, Vergari S. Neural networks and support vector machine algorithms for automatic cloud classification of whole-sky ground-based images. IEEE Geosci Remote Sens Lett. 2015;12(3):666-670. https://doi.org/10.1109/LGRS.2014.2356616

21. Xia M, Lu W, Yang J, Ma Y, Yao W, Zheng Z. A hybrid method based on extreme learning machine and k-nearest neighbor for cloud classification of ground-based visible cloud image. Neurocomputing. 2015;160:238-249. https://doi.org/10.1016/j.neucom.2015.02.022

22. Kazantzidis A, Tzoumanikas P, Bais A, Fotopoulos S, Economou G. Cloud detection and classification with the use of whole-sky groundbased images. Atmos Res. 2012;113:80-88. https://doi.org/10.1016/j. atmosres.2012.05.005

23. Jayadevan VT, Rodriguez JJ, Cronin AD. A new contrast-enhancing feature for cloud detection in ground-based sky images. J Atmos Oceanic Tech. 2015;32(2):209-219. https://doi.org/10.1175/JTECHD-14-00053.1

24. Ineichen $P$, Perez R. A new airmass independent formulation for the Linke turbidity coefficient. Sol Energy. 2002;73(3):151-157. https:// doi.org/10.1016/S0038-092X(02)00045-2

25. Hanrieder N, Sengupta M, Xie Y, Wilbert S, Pitz-Paal R. Modeling beam attenuation in solar tower plants using common DNI measurements. Sol Energy. 2016;129:244-255. https://doi.org/10.1016/ j.solener.2016.01.051

26. Wilbert S, Kleindiek S, Nouri B, et al. Uncertainty of rotating shadowband irradiometers and Si-pyranometers including the spectral irradiance error. AIP Conf Proc. 2016;1734(1): 150009. https://doi. org/10.1063/1.4949241

27. Calbó J, Long CN, González J-A, Augustine J, McComiskey A. The thin border between cloud and aerosol: sensitivity of several ground based 
observation techniques. Atmos Res. 196:248-260. https://doi.org/ 10.1016/j.atmosres.2017.06.010

28. Hay JE, McKay DC. Estimating solar irradiance on inclined surfaces: a review and assessment of methodologies. Int J Sol Energy. 1985;3(4-5):203-240. https://doi.org/10.1080/01425918508914395

29. Yoshida S, Ueno S, Kataoka N, Takakura H, Minemoto T. Estimation of global tilted irradiance and output energy using meteorological data and performance of photovoltaic modules. Sol Energy. 2013;93:90-99. https://doi.org/10.1016/j.solener.2013.04.001

30. Gueymard CA, Ruiz-Arias JA. Extensive worldwide validation and climate sensitivity analysis of direct irradiance predictions from 1-min global irradiance. Sol Energy. 2016;128:1-30. https://doi.org/10.1016/ j.solener.2015.10.010

31. Kuhn P, Wilbert S, Prahl C, et al. Shadow camera system for the generation of solar irradiance maps. Sol Energy. 2017;157(Supplement C):157-170. https://doi.org/10.1016/j.solener.2017.05.074

32. Kuhn, P., S. Wilbert, C. Prahl, A. Kazantzidis, L. Ramírez, L. Zarzalejo, L. Vuilleumier, P. Blanc, and R. Pitz-Paal, Validation of nowcasted spatial DNI maps, in DNICast, Deliverable 4.1Validation of nowcasted spatial DNI maps, German Aerospace Center (DLR), available online: http:// www.dnicast-project.net/

33. Kuhn, P., S. Wilbert, D. Schüler, C. Prahl, T. Haase, L. Ramirez, L. Zarzalejo, A. Meyer, L. Vuilleumier, P. Blanc, J. Dubrana, A. Kazantzidis, M. Schroedter-Homscheidt, T. Hirsch, and R. Pitz-Paal. Validation of spatially resolved all sky imager derived DNI Nowcasts. in AIP conference proceedings (SolarPACES) 2017, http://aip.scitation.org/ doi/abs/10.1063/1.4984522
34. Bernecker D, Riess C, Angelopoulou E, Hornegger J. Continuous short-term irradiance forecasts using sky images. Sol Energy. 2014;110:303-315. https://doi.org/10.1016/j.solener.2014.09.005

35. Schmidt T, Kalisch J, Lorenz E, Heinemann D. Evaluating the spatio-temporal performance of sky-imager-based solar irradiance analysis and forecasts. Atmos Chem Phys. 2016;16(5):3399-3412. https://doi.org/10.5194/acp-16-3399-2016

36. Fu C-L, Cheng H-Y. Predicting solar irradiance with all-sky image features via regression. Sol Energy. 2013;97:537-550. https://doi.org/ 10.1016/j.solener.2013.09.016

37. Wang G, Kurtz B, Kleissl J. Cloud base height from sky imager and cloud speed sensor. Sol Energy. 2016;131:208-221. https://doi.org/ 10.1016/j.solener.2016.02.027

38. Marcos J, Marroyo L, Lorenzo E, Alvira D, Izco E. Power output fluctuations in large scale PV plants: one year observations with one second resolution and a derived analytic model. Prog Photovolt Res Appl. 2011;19(2):218-227. https://doi.org/10.1002/ pip.1016

How to cite this article: Kuhn P, Nouri B, Wilbert S, et al. Validation of an all-sky imager-based nowcasting system for industrial PV plants. Prog Photovolt Res Appl. 2017;1-14. https://doi.org/10.1002/pip.2968 